\title{
Video Article \\ Use of MRI-ultrasound Fusion to Achieve Targeted Prostate Biopsy
}

\author{
Rajiv Jayadevan ${ }^{1}$, Steve Zhou ${ }^{1}$, Alan M. Priester ${ }^{1}$, Merdie Delfin ${ }^{1}$, Leonard S. Marks ${ }^{1}$ \\ ${ }^{1}$ Department of Urology, University of California Los Angeles
}

Correspondence to: Rajiv Jayadevan at rjayadevan@mednet.ucla.edu

URL: https://www.jove.com/video/59231

DOI: doi:10.3791/59231

Keywords: Medicine, Issue 146, prostate, fusion, MRI, fusion biopsy, targeted biopsy, prostate cancer

Date Published: 4/9/2019

Citation: Jayadevan, R., Zhou, S., Priester, A.M., Delfin, M., Marks, L.S. Use of MRI-ultrasound Fusion to Achieve Targeted Prostate Biopsy. J. Vis. Exp. (146), e59231, doi:10.3791/59231 (2019).

\section{Abstract}

Here, we present a protocol to perform targeted prostate biopsy using a magnetic resonance imaging-ultrasound (MRI/US) fusion system. Prostate cancer has traditionally been diagnosed via transrectal ultrasound (TRUS) biopsy. Though considered the gold standard, TRUS is unable to visualize most prostate cancer lesions and therefore requires sampling of the entire prostate. This biopsy method often undergrades prostate cancer and fails to detect up to $35 \%$ of cancers on initial biopsy. Prostate MRI has been shown to have excellent sensitivity in the detection of cancerous lesions, and advancements in MRI technology during the last decade have led to the development of targeted biopsy. In targeted biopsy, a software platform overlays MRI data onto live TRUS images to create a fused MRI/US three-dimensional model of the prostate. Regions suspicious for malignancy on MRI are contoured by a radiologist, uploaded into the fusion system, and then displayed within the live MRI/US fused model. The urologist is then able to directly biopsy these targets. When compared to conventional TRUS biopsy, MRI/US fusion technology has been demonstrated to improve the detection of clinically significant cancer while reducing insignificant cancer detection. This technology, therefore, has the potential to diagnose prostate cancer primarily in men who would benefit from treatment.

\section{Video Link}

The video component of this article can be found at https://www.jove.com/video/59231/

\section{Introduction}

Prostate cancer is the second most common cancer in American men, with nearly 165,000 cases diagnosed in $2018^{1}$. The majority of these cases were diagnosed via transrectal ultrasound (TRUS)-guided biopsy, a methodology that was first developed in the 1960s before gaining widespread acceptance in the $1980 \mathrm{~s}^{2}$. In TRUS biopsy, the clinician typically performs a sextant biopsy, systematically sampling the base, middle, and apex of each hemigland ${ }^{3}$. Despite being long considered the gold standard for diagnosis, TRUS biopsy has several shortcomings. Because ultrasound usually fails to visualize cancer, a TRUS biopsy is performed by systematically sampling all parts of the prostate, rather than aiming at individual targets (Figure 1). Thus, TRUS biopsy is "blind" and under-grading occurs in as many as $46 \%$ of patients, and up to $35 \%$ of cancers are undetected on the first TRUS biopsy ${ }^{4,5}$.

Prostate magnetic resonance imaging (MRI), reported as early as 1983 , has revolutionized prostate cancer diagnosis during the past decade ${ }^{6}$. Multiparametric MRI (mpMRI) combines T1 and T2 contrast imaging with diffusion weighted imaging (DWI) and dynamic contrast enhancement (DCE) to create both an anatomic and functional assessment of the gland ${ }^{7}$. This combined multiparametric imaging modality facilitates tumor visualization and has been shown to have superior ability to detect prostate cancer. As compared to TRUS biopsy that has a sensitivity of approximately $60 \%$, mpMRI has been demonstrated to have sensitivity as high as $96 \%$ in the detection of lesions that are later confirmed to harbor prostate cancer ${ }^{8,9,10,11}$. To increase standardization of mpMRI interpretation, the European Society of Urogenital Radiology developed the Prostate Imaging-Reporting and Data System (PI-RADS) for regions of interest (ROI) that are suspicious for prostate cancer ${ }^{12}$. ROIs are graded on a five-point Likert scale, where a score of 1 has very low risk of malignancy and a score of 5 is considered a high-risk lesion. ROIs classified as Grade 3 or above are often pursued during prostate biopsy.

Advancements in MRI technology have led to the development of targeted prostate biopsy, which is facilitated by MRI-ultrasound (MRI/US) fusion. In this modality, a software platform overlays mpMRI data onto live transrectal ultrasound images and creates a fused three-dimensional (3D) model, enabling the operator to visualize an MRI-detected ROI in real time on a monitor. Once both the MRI and US are registered, ROIs seen on the MRI image are transferred to the ultrasound image. These ROls may then be individually targeted, known as the "targeted biopsy". The trajectory of each needle and biopsy core location are tracked with a high degree of accuracy and registered within the software system (Figure 2). This allows the clinician to resample a target within $3 \mathrm{~mm}$ at any follow-up biopsy session ${ }^{13,14}$. Biopsy tracking is particularly useful in active surveillance programs in that foci of low-risk cancer may be reliably monitored for pathologic progression over time.

During the past decade, several MRI/US fusion devices have been developed for commercial use, and several studies have investigated the efficacy of this biopsy method. Two large prospective trials recently demonstrated the value of MRI/US fusion technology in the diagnosis of prostate cancer ${ }^{15,16}$. In both studies, guidance by MRI/US fusion was compared to standard sextant TRUS biopsy in men with MRI-visible lesions. When MRI/US fusion was used, targeted biopsy detected more cases of clinically significant prostate cancer than TRUS biopsy alone, and in one of the studies the new method detected fewer cases of insignificant prostate cancer ${ }^{15}$. With reduced detection of clinically insignificant 
cancer, guided biopsy can spare many patients the emotional distress of a cancer diagnosis as well as the morbidity associated with further prostate biopsies. Patients harboring intermediate or high-risk prostate cancer are likely to be diagnosed via guided biopsy and can be referred for treatment accordingly.

UCLA launched its MRI/US fusion biopsy program in early 2009 with the release of the first Food and Drug Administration (FDA)-approved fusion biopsy platform. Several platforms have now been developed and are available internationally. Each uses proprietary software and hardware to merge MRI and US data in real time to enable targeted biopsy. Table 1 presents data for several of the most commonly used fusion systems ${ }^{17}$. The UCLA experience is primarily with the Artemis and UroNav systems, via which the vast majority of fusion biopsies in the United States are currently performed.

Performed in the clinic under local anesthesia, this new biopsy method is rapidly gaining adoption for the diagnosis and surveillance of prostate cancer. Herein we provide a technical protocol for performing targeted prostate biopsy via MRI/US fusion.

\section{Protocol}

When used in a research capacity, the protocol provided below strictly follows guidelines established by the UCLA human research ethics committee.

NOTE: The methods described here are those used at UCLA for performing targeted biopsy of the prostate using the Artemis system. All patients undergoing fusion biopsy have had mpMRI of the prostate, which has been interpreted by an experienced uro-radiologist who has read over 3,500 prostate MRIs. Lesions visible on mpMRI have been classified as PI-RADS 1-5, with PI-RADS 3-5 lesions selected for targeted biopsy. Prior to the procedure, MRI images are uploaded to software for prostate and target contouring by the radiologist. All patients undergoing targeted biopsy also undergo systematic biopsy, guided by a template generated by the fusion device software. If no discrete targets are seen on MRI, only software-guided systematic biopsy is performed. Patients with suspected or previously-diagnosed prostate cancer are considered eligible for MRI/US fusion biopsy. Patients with bleeding diathesis or inability to tolerate biopsy without sedation are considered ineligible.

\section{Machine Initiation and Biopsy Plan Selection}

1. Power on the workstation computer and the workstation cart.

2. Enter the new patient's information or select a patient if the patient has already been registered within the software system. Import the MRI data that have been uploaded via the device's contouring software.

3. Select biopsy plan type (e.g., MRI-TRUS fusion biopsy, revisit biopsy, or systematic biopsy). Select all three biopsy plan types to allow for biopsy of new targets, resampling of prior locations, and the performance of a systematic sextant biopsy. The fusion system prompts the physician to select either 6 or 12 biopsy locations to be proposed if choosing systematic biopsy (i.e., either 1 or 2 cores from each anatomic sextant).

NOTE: In the UCLA practice, all patients undergoing targeted biopsy also undergo simultaneous systematic biopsy. Twelve systematic biopsy cores rather than six are usually taken for the sake of thoroughness.

\section{Patient Preparation}

1. Prescribe an enema and direct the patient to use it the morning of biopsy for rectal vault cleaning.

2. Administer antibiotics one hour prior to the start of the procedure. Administer either fluoroquinolones, first, second, or third generation cephalosporins, or aminoglycosides as recommended by the American Board of Urology.

NOTE: It is critical to review the local antibiogram when selecting the antibiotic to be used. At UCLA, $1 \mathrm{~g}$ of Ertapenem is administered intramuscularly one hour prior to the procedure. This decision was made based on the UCLA antibiogram. There have been no post-biopsy septic episodes for the last 1,500 MRI-US fusion biopsies performed.

3. Place the patient in the left lateral decubitus position. Place the patient's back nearly parallel to the edge of the bed, with the patient's legs pulled toward the chest to provide maximum range of motion for the biopsy device's tracker arm. Ensure that the patient's buttocks are positioned slightly off the edge of the bed.

4. Prepare the patient's anus. Soak a sponge stick in the preferred antiseptic solution and swab the perineum and anus, starting away from the anus and moving toward the anus.

5. Perform a digital rectal examination. Insert a gloved and lubricated index finger into the rectum and direct anteriorly to palpate the prostate. NOTE: If a nodule or induration is palpated, biopsy of the abnormality should be performed.

\section{TRUS Probe Preparation}

1. Attach the needle guide to TRUS probe.

2. Apply ultrasound jelly directly to a clean TRUS probe.

NOTE: At UCLA, all TRUS probes are disinfected via an automated system that uses vaporized hydrogen peroxide solution.

3. Fit a condom cover onto TRUS probe directly over the ultrasound jelly and secure it in place with a rubber band.

\section{Administer Periprostatic Nerve Block}

1. Gently insert the lubricated end-fire TRUS probe into the patient's rectum. Advance the probe until a two-dimensional (2D) transverse view of the prostate is clearly visible on the monitor and adjust the probe until the approximate center of the prostate is visible.

2. Obtain an estimated prostate volume by measuring prostate height, width, and length. Calculate prostate specific antigen (PSA) density if desired by dividing PSA value by prostate volume. 
3. Turn on the on-screen biopsy guide in order to visualize needle trajectory.

4. Rotate and advance the probe until the junction between the prostate and seminal vesicle is visualized, representing the area where the prostatic neurovascular bundle enters the gland.

5. Insert a $22 \mathrm{G}$ spinal needle through the needle guide channel on the TRUS probe. Advance the needle into the junction between prostate and seminal vesicle.

6. Infiltrate this space with $10 \mathrm{cc}$ of $1 \%$ lidocaine, creating an ultrasonic wheal.

NOTE: Correct infiltration will cause a separation of the seminal vesicles and prostate from the rectal wall.

7. Readjust the TRUS probe to administer the periprostatic nerve block on the contralateral side. Wait 1 min for adequate anesthesia to be achieved.

\section{Dock the Tracking Arm}

1. Adjust the TRUS probe such that the prostate's greatest diameter is visible in transverse orientation.

2. Position the workstation cart next to the patient in order to visualize the workstation screens while also observing the patient.

3. Ensure that the tracking arm is in the "park" position. Place the two tracking arms approximately $90^{\circ}$ from each other.

4. Unlock the tracker arm and position it directly underneath the TRUS probe while holding the TRUS probe in place within the patient's rectum.

5. Lift the tracker arm to place the TRUS probe into the cradle of the tracking arm and secure the clasp. The TRUS probe is now secure.

6. Lock the stabilizing arm.

\section{Three-dimensional Image Acquisition}

1. Slowly rotate the TRUS probe clockwise along its long axis for approximately $200^{\circ}$.

NOTE: The ultrasound system will acquire 2D images and reconstruct them to create a 3D ultrasound model that is stored within the software platform.

2. Outline the prostate by placing 6-8 green digital markers along its border in both the transverse and sagittal images displayed on the workstation screen. A 3D reconstruction of the prostate will then be created by the software and displayed.

3. Review the 3D ultrasound model of the prostate to ensure the prostate is visible in all slices.

4. Refine the proposed boundaries of the prostate on the 3D ultrasound reconstruction by clicking the correct prostate boundary on the grayscale image. Only perform refinement if there is a discrepancy between the green contour and the true boundary of the prostate.

\section{MRI Registration}

1. Perform rigid registration in the saggital orientation of the MRI, which will be presented on the display screen. Select two landmarks on the MR image (eg: superior-most point and inferior-most point of the prostate along the rectum wall) and place a digital marker on each. Place two digital markers on corresponding landmarks on the displayed ultrasound image.

2. Perform rigid registration in the transverse orientation. Again, select two landmarks on the MRI image (e.g., anterior-most point and posteriormost point of the prostate) and place a digital marker on each. Place two digital markers on corresponding landmarks on the displayed ultrasound image. NOTE: Elastic registration is automatically performed after selecting the "Next" button on the display screen.

\section{Target Acquisition}

NOTE: Contoured targets from the mpMRI as well as digital markers denoting a template for systematic biopsy are now superimposed onto the 3D prostate model created during the acquisition step.

1. Select the first ROI to be biopsied.

2. Depress the clutch near the tracker arm handle to release the tracker arm braking system. Gently move the tracker arm toward the desired target. Once the yellow digital marker is in the desired location over the target - now highlighted in red - release the clutch to reengage the brakes on the tracker arm. The tracker arm is now secure in space.

3. Disengage the rotation brake by pushing the lever just left of the tracker arm handle forward. Gently rotate the tracker arm to align the yellow digital marker until it is superimposed onto the desired target. Pull the lever to reengage the rotational brakes.

\section{Motion Compensation}

1. Prior to each biopsy, assess whether the live ultrasound images remain registered within the ultrasound reconstruction. If the border of the prostate on live ultrasound is contained within the series of green digital markers, proceed to section 10. If the green digital markers no longer correctly demarcate the border of the prostate, carry out motion compensation.

1. Select the motion compensation option on the monitor.

2. Choose three landmarks on the 3D prostate reconstruction and place a digital marker on each. Place digital markers on the corresponding landmarks on the live ultrasound view of the prostate in order to bring the 3D model back into registration with the live ultrasound.

\section{Prostate Biopsy and Needle Recording}

1. Place the $18 \mathrm{G}$ biopsy gun into the needle guide mounted on the TRUS probe. 
2. While observing the monitor, advance the biopsy needle toward the red bowtie-shaped visual aid that is displayed over the live ultrasound image. Advance the needle tip to intersect the middle of the bowtie-shaped marker.

3. Depress the footswitch pedal to begin recording the sequence of $2 \mathrm{D}$ ultrasound images for 3D biopsy location recording, which will be used to mark the site of the completed biopsy and can be revisited at a later time for future review.

4. Fire the biopsy needle by depressing the button on the biopsy gun. Pay careful attention to the streak of the needle on the ultrasound.

5. Release the footswitch to stop needle recording and then remove the biopsy gun from the needle guide.

6. Hand the biopsy gun to the assistant. Let the assistant open the sheath and deposit the biopsy core into its own labeled specimen cup containing $10 \%$ buffered formalin.

\section{Needle Segmentation}

1. Review the needle trajectory recording and observe the white streak the needle creates when viewed by ultrasound. Compare the streak on the recorded ultrasound frames to the red needle auto-segmentation line that appears as an overlay within the pop-up window. Save the needle auto-segmentation if it is accurate in order to permanently store the location data for this biopsy core.

2. If needle auto-segmentation is inaccurate, correct the trajectory manually.

1. Use the toggle arrows to scroll through the needle recording sequence until the needle image frame is found.

2. Define the needle tip and trajectory by marking the points corresponding to beginning and end of the needle streak. Click on the tip of the streak to define the needle tip and click on the bottom of the streak to define the needle trajectory.

3. Save this needle segmentation or readjust once again.

NOTE: Saving will permanently store the location data for this biopsy core and overlay it onto the 3D model.

\section{Remaining Tissue Extraction}

1. Repeat sections 8-11 until tissue is extracted from all desired locations.

2. Determine the number of cores to obtain from each mpMRI-recognized ROI. In order to ensure that each ROI is well-sampled, consider obtaining cores at set intervals (e.g., every $3 \mathrm{~mm}$ ), or from both the center and the periphery.

\section{Conclude the Biopsy Session}

1. Unlock the stabilizer arm. Gently remove the TRUS probe from the patient's rectum. Apply pressure with gauze pads for 5 min in order to facilitate hemostasis.

\section{Representative Results}

Between 2009 and 2015, 1,042 men underwent MRI/US fusion biopsy at UCLA for either elevated PSA, abnormal digital rectal exam, or for confirmation of low-risk prostate cancer in patients considering active surveillance. Subjects underwent mpMRI of the prostate with a 3 Tesla magnet prior to biopsy. ROls were graded on a 1-5 Likert scoring system based on suspicion of malignancy that was developed at UCLA prior to the establishment of PI-RADS. Similar to PI-RADS, the UCLA score is based on T2-weighted imaging, DWI, and DCE. Regions graded as "1" had normal T2-weighted imaging, normal DCE, and ADC on DWI of $>1.2 \times 10^{-3} \mathrm{~mm}^{2} / \mathrm{s}$, while regions graded as " 5 " had a dark nodule with mass effect on T2-weighted imaging, profoundly abnormal DCE, and ADC on DWI of $<0.6 \times 10^{-3} \mathrm{~mm}^{2} / \mathrm{s}$.

Following mpMRI, the MRI images were transferred to the fusion biopsy system's contouring software in which ROI contouring was performed, and then sent to the MRI/US fusion biopsy device. This system was used to obtain targeted cores from ROls (if present). All patients underwent a 12-core systematic biopsy using a template generated by the fusion system regardless of whether targeted biopsy was performed. All sextants were sampled during systematic biopsy, including those that contained ROls. The primary outcome was the detection of clinically significant prostate cancer, defined as Gleason score $\geq 7$. The detection of clinically significant prostate cancer was compared between the different fusion biopsy strategies in patients with at least $1 \mathrm{ROI}$ of $\geq$ grade 3 . The biopsy strategies compared were targeted biopsy, systematic biopsy, and the simultaneous performance of both targeted and systematic biopsy within the same session, known as the "combination biopsy."

Figure 3 demonstrates the performance of combination biopsy compared to targeted biopsy and systematic biopsy. Among all patients, 825 patients had at least one ROI classified as grade 3 or higher. For maximum ROI grade, 435 patients had a grade 3 lesion, 301 had a grade 4 lesion, and 89 had a grade 5 lesion. Among the 825 patients with an ROI $\geq$ grade 3 , combination biopsy had the greatest detection rate for clinically significant cancer. While 289 cases of clinically significant disease were detected via combination biopsy, 229 patients with clinically significant disease were identified using targeted biopsy alone and 199 were identified with systematic biopsy alone. Combination biopsy also identified a greater number of high-risk ( $\geq$ Gleason 8 ) prostate cancer cases, with 89 high-risk cases diagnosed with combination biopsy compared to 74 via targeted biopsy alone $(p<0.001)$ and 51 with systematic biopsy alone $(p<0.001)$. Of this group, 15 patients with high-risk disease would have otherwise been undiagnosed if only targeted biopsy was performed.

The identification of clinically significant prostate cancer was directly related to ROI grade. $80 \%$ of men with a grade 5 ROI had Gleason $\geq 7$ disease compared to $24 \%$ for men with grade 3 ROI. Combination biopsy also outperformed both targeted biopsy and systematic biopsy for all grades of ROI (Figure 4). 


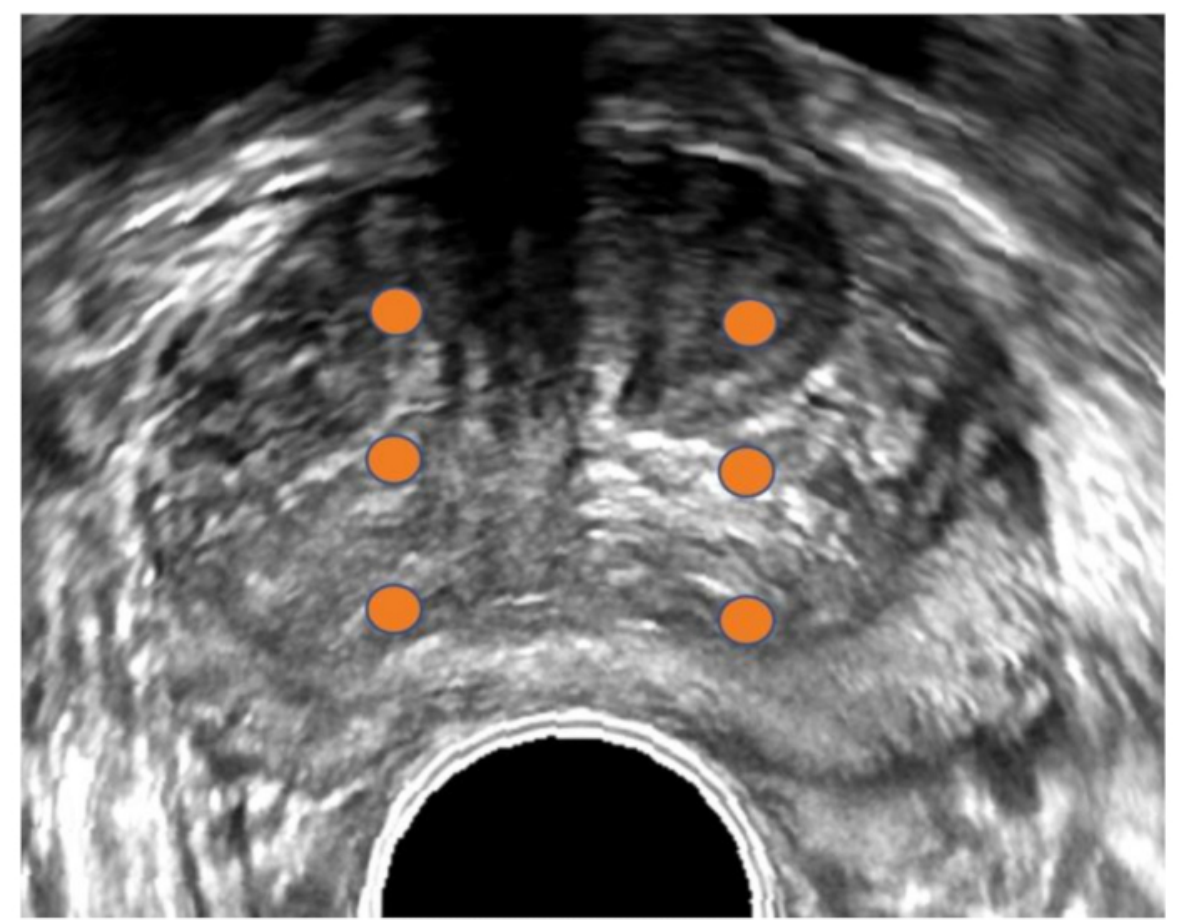

Figure 1: Transrectal ultrasound image of prostate. Conventional transrectal ultrasound (TRUS) image of prostate in transverse orientation. Orange dots demarcate sextant biopsy plan. The TRUS method is usually blind to tumor location since most tumors are not visible on ultrasound. Please click here to view a larger version of this figure.
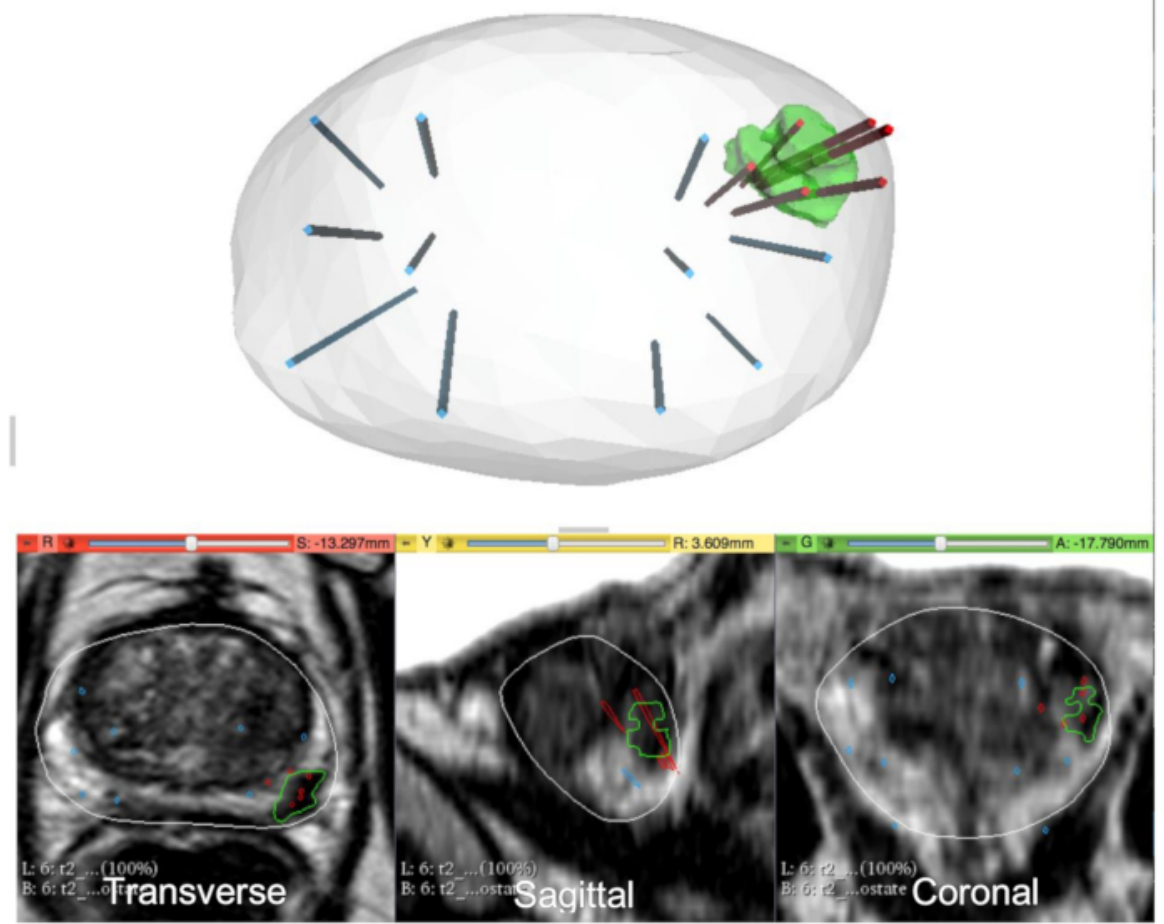

Figure 2: 3D reconstruction of prostate. 3D reconstruction of the prostate (upper panel) and MRI with visible region of interest (ROI) shown in transverse, sagittal, and coronal views (lower panels). The ROI is shown in green (upper) and contoured in green (lower). Cores positive for malignancy are shown in red. Other cores shown in blue are negative, making this patient a possible candidate for focal therapy. Please click here to view a larger version of this figure. 


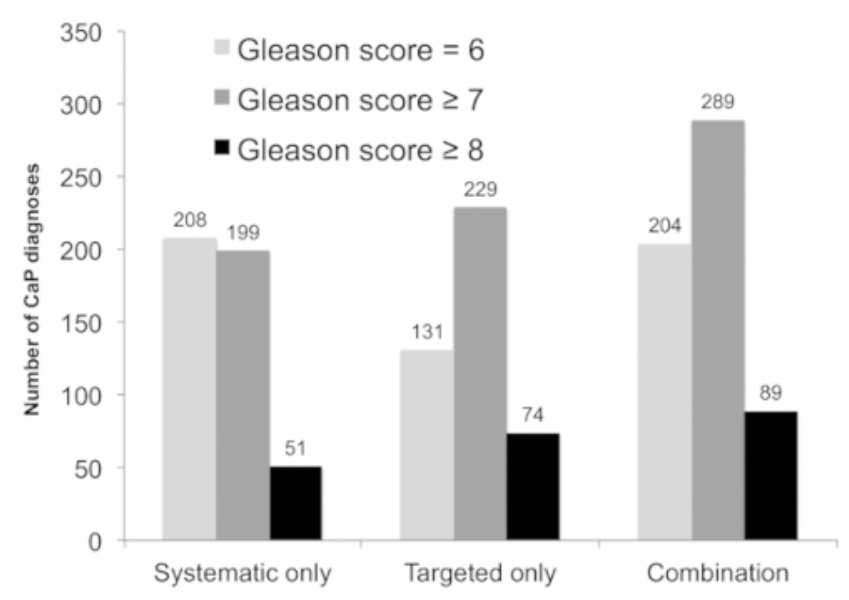

Figure 3: Diagnostic performance of systematic biopsy, targeted biopsy, and combined approach among patients whose mpMRI revealed at least one $\mathrm{ROI}$ of grade $\geq 3(n=\mathbf{8 2 5})$. The number of patients diagnosed with prostate cancer $(\mathrm{CaP} ; \mathrm{y}$-axis) versus the biopsy strategy (x-axis) is shown. Combining targeted and systematic biopsies resulted in the detection of 60 clinically significant cancers undetected by either alone (light gray, $p<0.001$ versus systematic and targeted alone), and an additional 15 high-risk cases (black, $p<0.001$ versus systematic and targeted approach). This figure is adapted with permission from Filson et al. ${ }^{19}$. Please click here to view a larger version of this figure.

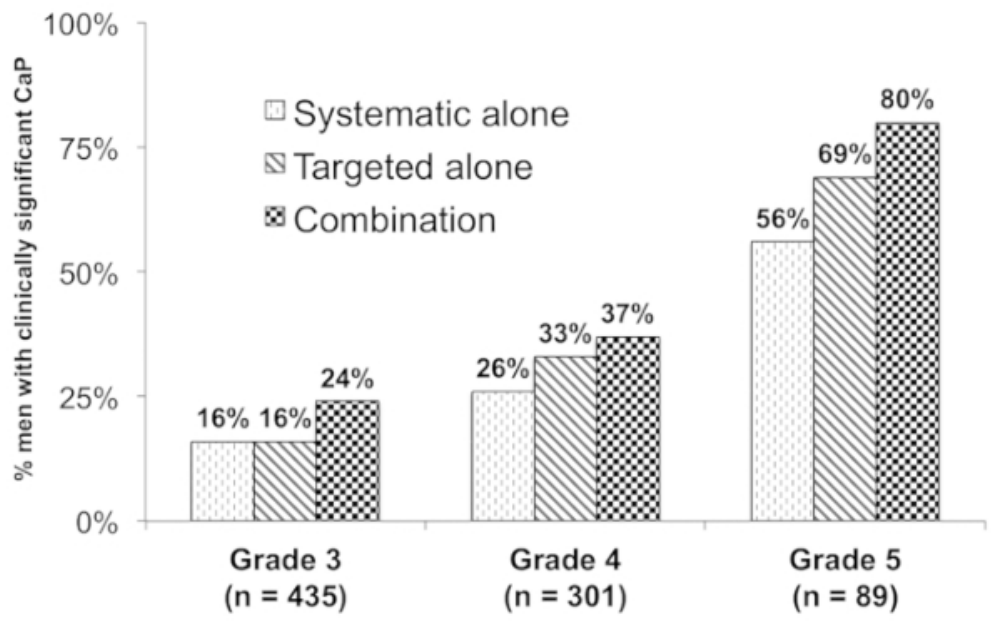

Maximum ROI Grade

Figure 4: Relationship between the ROI grade and presence of cancer. This figure shows the proportion of patients with $\geq 1 \mathrm{ROI}$ on MRI ( $\mathrm{n}$ $=825$ ) with a diagnosis of clinically significant $\mathrm{CaP}(\mathrm{n}=289,35 \%)$ (y-axis) stratified by ROI grade (x-axis). Combination biopsy (black checked bars) outperformed systematic biopsy (dark diagonal bars) and targeted biopsy (light hatched bars) across all ROI grades ( $p<0.001$ ). Overall, $80 \%$ of patients with a grade $5 \mathrm{ROI}$ had clinically significant CaP (versus $24 \%$ grade 3 ROI, odds ratio 9.05 , 95\% confidence interval $4.96-$ 16.50). This figure is adapted with permission from Filson et al. ${ }^{19}$. Please click here to view a larger version of this figure. 


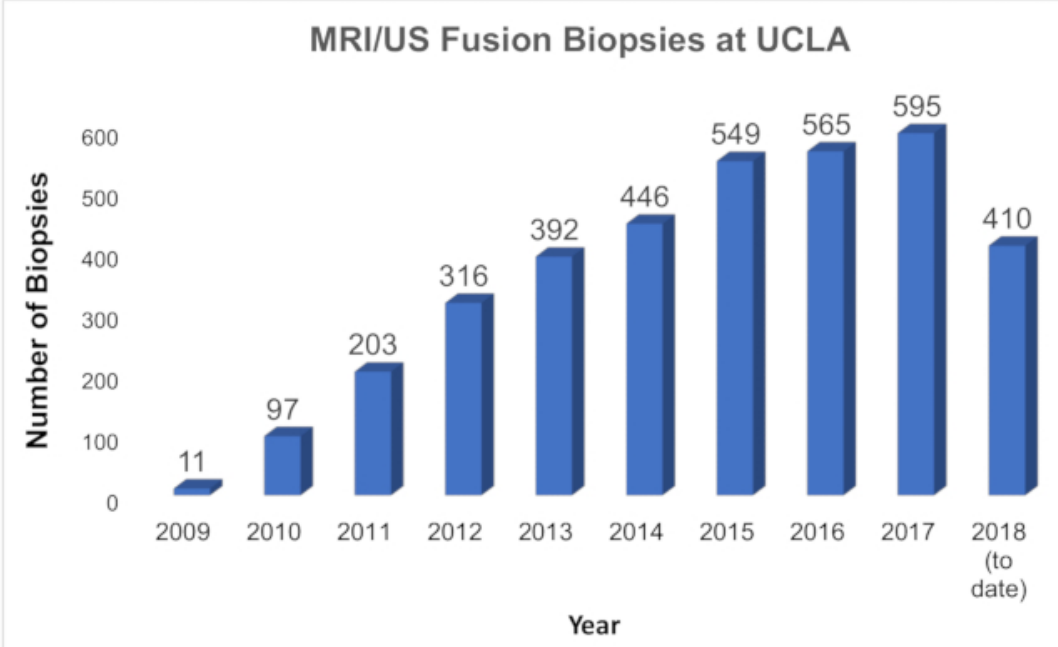

Figure 5: Growth of MRI/US fusion biopsies at UCLA. Chart showing the number of MRI/US fusion biopsies performed annually at UCLA since inception of the program in 2009. At UCLA, the new technology is used for first-time biopsy, for repeat biopsy, and serially for men in active surveillance. Please click here to view a larger version of this figure.

\begin{tabular}{|c|c|c|c|c|}
\hline $\begin{array}{l}\text { Artemis } \\
\text { (Eigen, USA) }\end{array}$ & $\begin{array}{l}\text { Position-Encoded Joints on } \\
\text { Robotic Arm }\end{array}$ & $\begin{array}{l}\text { In/out and rotational } \\
\text { movement only (fixed to } \\
\text { mechanical arm) }\end{array}$ & Transrectal & \begin{tabular}{|l} 
Robotic arm minimizes \\
human error \\
Training required to learn \\
software and manual \\
manipulation of TRUS \\
biopsy via mechanical arm
\end{tabular} \\
\hline $\begin{array}{l}\text { BioJet } \\
\text { (GeoScan Medical, USA) }\end{array}$ & $\begin{array}{l}\text { Position-Encoded Joints on } \\
\text { Robotic Arm }\end{array}$ & $\begin{array}{l}\text { In/out and rotational } \\
\text { movement only }\end{array}$ & Transrectal or transperineal & $\begin{array}{l}\text { TRUS-probe mounted to } \\
\text { angle-sensing mechanical } \\
\text { arm that exports information } \\
\text { on probe position to } \\
\text { workstation. }\end{array}$ \\
\hline $\begin{array}{l}\text { BiopSee } \\
\text { (Pi Medical, Greece) }\end{array}$ & $\begin{array}{l}\text { Position-Encoded Joints on } \\
\text { Robotic Arm }\end{array}$ & $\begin{array}{l}\text { In/out and rotational } \\
\text { movement only (fixed to } \\
\text { mechanical arm) }\end{array}$ & Transperineal & $\begin{array}{l}\text { Biopsy setup similar to } \\
\text { brachytherapy; } \\
\text { Diagnostic setup can } \\
\text { potentially be used for } \\
\text { treatment setup. } \\
\text { TRUS probe guides } \\
\text { transperineal biopsies }\end{array}$ \\
\hline $\begin{array}{l}\text { Real-Time Virtual } \\
\text { Sonography (Hitachi, } \\
\text { Japan) }\end{array}$ & $\begin{array}{l}\text { Electromagnetic Field } \\
\text { Generator for co- } \\
\text { registration of MRI and US } \\
\text { images }\end{array}$ & Freely movable by hand & Transrectal or transperineal & $\begin{array}{l}\text { Primarily used in Japan; } \\
\text { little studied elsewhere }\end{array}$ \\
\hline $\begin{array}{l}\text { UroNav } \\
\text { (Invivo, USA) }\end{array}$ & $\begin{array}{l}\text { Electromagnetic Field } \\
\text { Generator for co- } \\
\text { registration of MRI and US } \\
\text { images }\end{array}$ & Freely movable by hand & Transrectal & $\begin{array}{l}\text { First office-based fusion } \\
\text { platform on market } \\
\text { Familiar freehand TRUS } \\
\text { approach }\end{array}$ \\
\hline Urostation (Koelis, France) & $\begin{array}{l}\text { Software image-based } \\
\text { tracking } \\
\text { (3D US volume elastically } \\
\text { fused to 3D MR image in } \\
\text { real time) }\end{array}$ & Freely movable by hand & Transrectal & $\begin{array}{l}\text { Most common platform in } \\
\text { Europe } \\
\text { Relies purely on 3D TRUS } \\
\text { image tracking without any } \\
\text { beam-tracking external } \\
\text { hardware. }\end{array}$ \\
\hline
\end{tabular}

Table 1: MRI/US fusion devices commonly used in the United States and internationally. This table is adapted with permission from Elkhoury et al. ${ }^{17}$.

\section{Discussion}

The use of MRI/US fusion to guide prostate biopsy promises major advantages over traditional TRUS guidance in the diagnosis and surveillance of prostate cancer. TRUS biopsy is unique among image-guided biopsies in that tissue is not obtained from specific lesions, since the majority of prostate tumors are invisible on ultrasound ${ }^{18}$. The mpMRI has enabled urologists and radiologists to visualize and risk-stratify prostate lesions, 
helping to triage patients toward or away from biopsy. MRI/US fusion biopsy technology facilitates the sampling of MRI-visible lesions with great accuracy and reproducibility, and thus enhances the detection of clinically significant cancer compared with conventional TRUS biopsy.

The greatest value of MRI/US fusion technology lies within its ability to precisely project MRI-detected ROIs onto TRUS images for targeting. The accurate superimposition of MRI and TRUS images is therefore essential. Several critical steps performed during MRI/US fusion biopsy - either automatically or with clinician input - increase the accuracy of each biopsy. First and foremost is motion compensation, initiated by the clinician. Patient movement, even if slight, is unavoidable during an unsedated biopsy and can shift the overlay of MRI data onto TRUS images. The result is a "targeted biopsy" that misses its target. Motion compensation brings both MRI and TRUS images back into registration with one another. It is imperative to carry out motion compensation during MRI/US fusion biopsy in order to confirm absence of motion, and to frequently assess whether MRI and TRUS images remain accurately superimposed.

Compensation for other types of prostatic distortion is also performed during MRI/US fusion biopsy. Rigid registration, also performed by the clinician, corrects for prostate orientation differences based on patient positioning. These discrepancies occur because the mpMRI is acquired while the patient is in the supine position, while the 3D ultrasound is acquired while the patient is in the lateral decubitus position. Once rigid registration is complete, elastic registration is automatically performed by the software system. Elastic registration compensates for compression of the prostate from the TRUS probe. These advanced software-mediated features of MRI/US fusion enable the accurate sampling of ROls, thus enhancing cancer detection.

During targeted biopsy, care must be taken to ensure proper sampling of an ROI. Biopsy of ROls with the highest level of suspicion (as defined by PI-RADs v2) should be performed first, followed by ROls with lower level of suspicion, and finally the systematic sextant biopsy. This recommendation is based on the idea that tracking and image quality can decrease with each biopsy due to gland movement, prostate edema, or hematoma development. Accurate targeted biopsy is reliant on minimal anatomic discrepancy between prostate mpMRI and TRUS.

When sampling ROls, physicians should adhere to a biopsy strategy that maximizes the sampling of suspicious tissue while minimizing biopsy time and patient discomfort. One such strategy involves obtaining all cores from the center of the ROI. This method theoretically allows for tissue within an ROI to be sampled even if registration of MRI and TRUS is slightly skewed. Another strategy is to sample the center of the $\mathrm{ROI}$ as well regions in the periphery that may harbor a different grade of cancer. Larger ROls may require a greater number of cores to ensure appropriate sampling. At UCLA, the general guideline is to obtain 1 core of tissue every $3 \mathrm{~mm}$ of the longest axis. All biopsies directed at an ROI are considered to be targeted biopsies.

In recent years, an effort has been made to change prostate cancer screening methods in order to reduce overdiagnosis and overtreatment. The importance of diagnostic modalities that bear a high yield for clinically significant disease has increased. Because of the accuracy of MRI-US fusion for biopsy guidance, clinicians have sought greater implementation of this technology ${ }^{11,15,16}$. At UCLA, more than 3,500 fusion biopsies have been performed since the program's inception in 2009, an experience amongst the nation's largest (Figure 5). There has been continued growth of the program as the value of MRI/US fusion is increasingly recognized and new uses are developed. The ability of this technology to resample foci of cancer prompted the establishment of an active surveillance program based entirely on MRI/US fusion biopsy. Since 2009, more than 750 men with low-risk prostate cancer have been enrolled. Each patient undergoes MRI/US fusion biopsy every 1-2 years to resample both the original foci of cancer and systematically, other parts of the prostate. Patients with no pathologic progression remain on active surveillance and avoid radical treatment (and the possible adverse effects of such treatments). The diagnosis and surveillance of patients with MRI/US fusion technology leads to improved detection rates of those patients in need of treatment.

During initial biopsy using MRI/US fusion, systematic sampling is obtained along with targeted sampling of visible lesions. In this combination biopsy, both biopsy methods are performed using the MRI/US fusion system. The site of every biopsy core is recorded, both within and outside of MRI-visible lesions. The combination biopsy via the MRI/US fusion system allows detection of more clinically-significant prostate cancer than either method alone ${ }^{19}$. Why some lesions are undetected by MRI remains unclear. Some morphologies of prostate cancer, such as the aggressive cribiform variety, are not readily distinguishable from surrounding normal tissue on $\mathrm{MRI}^{20}$. Undetected cancer foci later discovered on whole mount pathology are often small, and lesions less than $0.5 \mathrm{cc}$ are frequently invisible on $\mathrm{MRI}^{21}$. Though small in volume these lesions may have relatively large surface areas, making them more likely to be detected via systematic biopsy than targeted biopsy. Systematic biopsy via the MRI/US fusion device may also be more beneficial than conventional TRUS systematic biopsy, since the software is able to propose biopsy locations that help to ensure an even sampling of the entire prostate. This enables the mapping of anatomic locations traditionally difficult to biopsy, such as the anterior prostate, and allows them to be included as part of systematic biopsy.

In addition to facilitating diagnosis, MRI/US fusion technology has the potential for use in the treatment of prostate cancer. Using fusion systems, lesions of cancer are accurately mapped and may then be targeted specifically for treatment. Known as "focal therapies", these types of selective treatment are currently used to treat low and intermediate-risk disease as alternatives to radical therapy. Recently, a phase I clinical trial on focal laser ablation of prostate cancer was performed using MRI/US fusion technology to facilitate accurate targeting of each intermediate-risk tumor ${ }^{22}$. Following treatment, patients were surveilled with $\mathrm{mpMRI}$ and had repeat MRI/US fusion targeted biopsy of the treated lesions to evaluate for persistent cancer. Evaluating the success of focal therapies would be challenging without the ability to accurately resample specific locations, as enabled by software tracking.

MRI/US fusion biopsy also has disadvantages. First and foremost, the cost to implement this system currently relegates it primarily to academic centers and large group practices. An MRI/US fusion device may cost upward of $\$ 150,000$ to purchase. Expenses are not limited to the actual device, however. In order to fully take advantage of the technology, patients must have access to both multiparametric prostate MRI and specially-trained uro-radiologists. Community-based practices - where the majority of patients in the United States are treated - will likely be unable to implement fusion technology due to current costs. Another impedance to the adoption of this technology is the time required to perform a fusion targeted biopsy. With the help of a trained assistant, each biopsy requires approximately 15 minutes, not including the time needed to upload and review MRI data. Two to three conventional TRUS biopsies may be completed during the same time period, which may serve as a financial disincentive for some urologists. In studies to date, the new technology is believed to be cost-effective because of the increased efficiency of cancer diagnosis. 
Men diagnosed with prostate cancer continue to be overtreated. MRI/US fusion biopsy technology has the potential to dramatically change the profile of men diagnosed with this disease. With less detection of insignificant disease and a greater yield of clinically significant tumors, we may soon diagnose mainly those who would benefit from surveillance and treatment.

\section{Disclosures}

Dr. Marks is a co-founder of Avenda Health, Inc.

\section{Acknowledgments}

The authors would like to acknowledge Rajesh Venkataraman (Eigen, Grass Valley, CA) for his technical support with this project.

\section{References}

1. Key Statistics for Prostate Cancer. www.Cancer.org. $1<\mathrm{https}: / /$ www.cancer.org/cancer/prostate-cancer/about/key-statistics.html> (2018).

2. Shiv, B. Y. et al. History of Prostate Biopsy. Urology News. 22 (2), 5-7 (2018).

3. Hodge, K. K., McNeal, J. E., Stamey, T. A. Ultrasound Guided Transrectal Core Biopsies of the Palpably Abnormal Prostate. The Journal of Urology. 142 (1), 66-70 (1989).

4. Noguchi, M., Stamey, T. A., McNeal, J. E., Yemoto, C. M. Relationship Between Systematic Biopsies and Histological Features of 222 Radical Prostatectomy Specimens: Lack of Prediction of Tumor Significance for Men With Nonpalpable Prostate Cancer. The Journal of Urology. 166 (1), 104-110 (2001).

5. Djavan, B. et al. Prospective evaluation of prostate cancer detected on biopsies 1, 2, 3 and 4: when should we stop? The Journal of Urology. 166 (5) 1679-1683 (2001).

6. Hricak, H. et al. Anatomy and pathology of the male pelvis by magnetic resonance imaging. American Journal of Roentgenology. 141 (6), 1101-1110 (1983).

7. Puech, P., Sufana lancu, A., Renard, B., Villers, A., Lemaitre, L. Detecting prostate cancer with MRI — why and how. Diagnostic and Interventional Imaging. 93 (4), 268-278 (2012).

8. Norberg, M. et al. The sextant protocol for ultrasound-guided core biopsies of the prostate underestimates the presence of cancer. Urology. $50(4), 562-566$ (1997).

9. Schoots, I. G. et al. Magnetic Resonance Imaging-targeted Biopsy May Enhance the Diagnostic Accuracy of Significant Prostate Cancer Detection Compared to Standard Transrectal Ultrasound-guided Biopsy: A Systematic Review and Meta-analysis. European Urology. 68 (3) 438-450 (2015).

10. Moldovan, P. C. et al. What Is the Negative Predictive Value of Multiparametric Magnetic Resonance Imaging in Excluding Prostate Cancer at Biopsy? A Systematic Review and Meta-analysis from the European Association of Urology Prostate Cancer Guidelines Panel. European Urology. 72 (2), 250-266 (2017).

11. Ahmed, H. U. et al. Diagnostic accuracy of multi-parametric MRI and TRUS biopsy in prostate cancer (PROMIS): a paired validating confirmatory study. The Lancet. 389 (10071), 815-822 (2017).

12. Barentsz, J. O. et al. ESUR prostate MR guidelines 2012. European Radiology. 22 (4), 746-757 (2012).

13. Natarajan, S. et al. Clinical application of a 3D ultrasound-guided prostate biopsy system. Urologic Oncology. 29 (3), $334-342$ (2011).

14. Marks, L., Young, S., Natarajan, S. MRI-ultrasound fusion for guidance of targeted prostate biopsy. Current Opinion in Urology. 23 (1), $43-50$ (2013).

15. Siddiqui, M. M. et al. Comparison of MR/Ultrasound Fusion-Guided Biopsy With Ultrasound-Guided Biopsy for the Diagnosis of Prostate Cancer. The Journal of the American Medical Association. 313 (4), 390-397 (2015).

16. Kasivisvanathan, V. et al. MRI-Targeted or Standard Biopsy for Prostate-Cancer Diagnosis (PRECISION study). New England Journal of Medicine. 378, 1767-1777 (2018).

17. Elkhoury, F. F., Simopoulos, D. N., Marks, L. S. MR-guided biopsy and focal therapy: new options for prostate cancer management. Current Opinion in Urology. 28 (2), 93-101 (2018).

18. Raja, J., Ramachandran, N., Munneke, G., Patel, U. Current status of transrectal ultrasound-guided prostate biopsy in the diagnosis of prostate cancer. Clinical Radiology. 61 (2), 142-153 (2006).

19. Filson, C. P. et al. Prostate cancer detection with magnetic resonance-ultrasound fusion biopsy: The role of systematic and targeted biopsies. Cancer. 122 (6), 884-892 (2016).

20. Truong, M. et al. A Comprehensive Analysis of Cribriform Morphology on Magnetic Resonance Imaging/Ultrasound Fusion Biopsy Correlated with Radical Prostatectomy Specimens. The Journal of Urology. 199 (1), 106-113 (2018).

21. Le, J. D. et al. Magnetic Resonance Imaging-Ultrasound Fusion Biopsy for Prediction of Final Prostate Pathology. The Journal of Urology. 192 (5), 1367-1373 (2014).

22. Natarajan, S. et al. Focal Laser Ablation of Prostate Cancer: Feasibility of Magnetic Resonance Imaging-Ultrasound Fusion for Guidance. The Journal of Urology. 198 (4), 839-847 (2017). 